Delft University of Technology

\title{
Low-Impedance PEDOT:PSS MEA Integrated in a Stretchable Organ-on-Chip Device
}

Waafi, Affan K.; Gaio, Nikolas; Quiros-Solano, William F.; Dijkstra, Paul; Sarro, Pasqualina M.; Dekker, Ronald

DOI

10.1109/JSEN.2019.2946854

Publication date

2020

Document Version

Final published version

Published in

IEEE Sensors Journal

Citation (APA)

Waafi, A. K., Gaio, N., Quiros-Solano, W. F., Dijkstra, P., Sarro, P. M., \& Dekker, R. (2020). Low-Impedance PEDOT:PSS MEA Integrated in a Stretchable Organ-on-Chip Device. IEEE Sensors Journal, 20(3), 11501157. [8864065]. https://doi.org/10.1109/JSEN.2019.2946854

\section{Important note}

To cite this publication, please use the final published version (if applicable).

Please check the document version above. 
Green Open Access added to TU Delft Institutional Repository

'You share, we take care!' - Taverne project

https://www.openaccess.nl/en/you-share-we-take-care

Otherwise as indicated in the copyright section: the publisher is the copyright holder of this work and the author uses the Dutch legislation to make this work public. 


\title{
Low-Impedance PEDOT:PSS MEA Integrated in a Stretchable Organ-on-Chip Device
}

\author{
Affan K. Waafi ${ }^{\circledR}$, Nikolas Gaio ${ }^{\circledR}$, William F. Quiros-Solano, Paul Dijkstra, \\ Pasqualina M. Sarro, Fellow, IEEE, and Ronald Dekker
}

\begin{abstract}
We present the first Organ-on-Chip equipped with a low-impedance Poly(3,4-ethylenedioxythiophene) polystyrene sulfonate (PEDOT:PSS) MicroElectrode Array (MEA). The novel device allows simultaneous mechanical stimulation with a stretchable PDMS membrane and electrical monitoring via the PEDOT:PSS MEA of multiple in vitro cell cultures. The surface area enhancement and the morphology of the PEDOT:PSS allows an increase of the charge injection per unit area at the electrode-electrolyte interface, resulting in significantly lower electrochemical impedance of the electrodes. In particular, at $1 \mathrm{kHz}$ the fabricated PEDOT-MEA electrodes show a reduction of the overall impedance up to 99.4 and $93.3 \%$ in comparison with benchmark TiN and Pt electrodes. The superior performance of PEDOT:PSS were also confirmed via Cyclic Voltammetry measurement, in which PEDOT:PSS showed a very large capacitive current, compared with the benchmark electrodes both in the forward and the reverse scans. The obtained results confirm the effectiveness of the proposed PEDOT:PSS coating, and introduce this material in the OOC field. Moreover, the quality and morphology of the fabricated PEDOT:PSS based electrodes were assessed via SEM imaging and Raman spectroscopy.
\end{abstract}

Index Terms—Organ-on-chip, microelectrode array, PEDOT:PSS, PDMS.

\section{INTRODUCTION}

$\mathbf{O}$ RGAN-ON-CHIPS (OOCs) are in vitro models, which replicate the minimal functional unit of an organ by combining advanced polymer chip technology with

Manuscript received May 5, 2019; revised October 3, 2019; accepted October 3, 2019. Date of publication October 10, 2019; date of current version January 17, 2020. This work was supported by the Electronic Components and Systems for European Leadership Joint Undertaking (ECSEL JU) InForMed Project under Grant 2014-2-662155. The work of $A$. Waafi was supported in part by the Lembaga Pengelola Dana Pendidikan (LPDP) Scholarship through the Indonesian Endowment Fund. The work of N. Gaio was supported in part by the InForMed Project. The work of W. F. Quiros-Solano was supported in part by the Instituto Tecnológico de Costa Rica. The associate editor coordinating the review of this article and approving it for publication was Prof. Sang-Seok Lee. (Affan K. Waafi and Nikolas Gaio contributed equally to this work.) (Corresponding author: Nikolas Gaio.)

A. K. Waafi, W. F. Quiros-Solano, and P. M. Sarro are with the Laboratory of Electronic Components, Technology, and Materials (ECTM), Else Kooi Lab (EKL), Delft University of Technology, 2628 CT Delft, The Netherlands.

N. Gaio is with the Laboratory of Electronic Components, Technology, and Materials (ECTM), Else Kooi Lab (EKL), Delft University of Technology, 2628 CT Delft, The Netherlands, and also with BIOND Solutions B.V., 2628 CT Delft, The Netherlands.

P. Dijkstra is with Philips Innovation Services, 5656 AE Eindhoven, The Netherlands.

R. Dekker is with the Laboratory of Electronic Components, Technology, and Materials (ECTM), Else Kooi Lab (EKL), Delft University of Technology, 2628 CT Delft, The Netherlands, and also with Philips Research, 5656 AE Eindhoven, The Netherlands.

Digital Object Identifier 10.1109/JSEN.2019.2946854 biology [1], [2]. They are microfabricated devices consisting of microfluidic components, e.g., channels, chambers, valves, and stretchable or porous membranes, on which cells are cultured [3], [4]. Higher control over phenomena such as cell growth, proliferation, differentiation, maturation and other cell processes are enabled by the dynamically controlled environment in the device [3].

Even though currently available OOCs allowed a deeper understanding of numerous cell processes [5], very frequently they lack the sensing mechanisms necessary to monitor these events, thus requiring the use of bulky conventional imaging techniques to identify and study the phenomena happening in the model [1]. Macroscopic platinum (Pt) wires have been manually inserted in soft-lithography-fabricated channels in order to perform transendothelial electrical resistance (TEER) measurements [6]. The fabrication of these devices and the integration of the electrodes heavily rely on several manual steps [6]. This approach strongly limits the miniaturization of the electrodes, and thus the integration of low-impedance Micro-Electrode Arrays (MEAs), an essential tool to perform in-situ monitoring of electrogenic tissues, such as neurons, muscles and cardiomyocytes, at a subcellular level [7]. Previous work has addressed this issue by combining commercially available MEAs with soft-lithography based microfluidic channels [8]-[10]. Even though this solution guarantees low-impedance MEAs, it does not allow for the integration 
of the electrodes in the stretchable membranes, a key element in OOCs.

To overcome these shortcomings, here we present an OOC equipped with a low-impedance MEA. To fabricate this device, the modular OOC platform known as Cytostretch is employed. This platform consists of freestanding and stretchable PDMS membrane fabricated on a silicon chip that can be equipped with different modules directly embedded in the membrane, such as pore arrays, strain gauges, microgrooves and MEAs. These features can be added through dedicated fabrication modules, to yield a chip with specific functionality for a particular OOC model as presented in [11]. The stretchable membrane itself is also an important feature to mechanically stimulate the cell culture [11].

In this work, instead of using only conventional electrode materials such as Pt, Titanium Nitride (TiN) and Gold (Au), known to provide high impedances, the Cytostretch MEA is coated with Poly(3,4-ethylenedioxythiophene) polystyrene sulfonate (PEDOT:PSS). This conductive organic polymer is best known for its low electrochemical impedance, high conductivity, transparency, biocompatibility and long-term stability in the biological environment [12]-[15]. These properties made PEDOT:PSS an attractive material choice in organic solar cells, light emitting devices, stretchable electronics and organic electrodes [7], [12]-[18]. By including PEDOT:PSS in the Cytostretch platform, a low-impedance PEDOT:PSS MEA is realized. This polymer guarantees sufficiently low noiseto-signal ratio during the detection of cellular action potential [7], a crucial requirement for several applications such as cardiotoxicity studies where the extracellular field potential generated by the cells' action potential needs to be studied in details to identify possible QT intervals prolongations often associated with safety-pharmacological risks [19]. Previous work has shown how PEDOT:PSS can be employed to bring the impedance from $330 \mathrm{k} \Omega$ down to $20 \mathrm{k} \Omega$ (at $1 \mathrm{kHz}$ ) on a commercially and non stretchable gold MEA [20]. This work is the first to focus on the integration of PEDOT:PSS MEA into a stretchable OOC device. Moreover, this work compares the resulting performance to more conventional electrode materials.

The use of PEDOT:PSS would also allow the integration of new sensors in the Cytostretch and the OOC field. PEDOT:PSS electrodes and in particular PEDOT:PSS based thin-film transistors have previously used to perform in vitro $\mathrm{pH}$ [21], humidity [22] and DNA [23] measurements, and to characterize the electrostatic interaction between the cells and the PEDOT:PSS surface [24].

The use of the Cytostretch platform for the development of this device, not only guarantees a successful integration of these PEDOT:PSS electrodes in an OOC, but also provides a conventional cleanroom-compatible micro-fabrication process, which avoids the often required labor-intensive fabrication steps used during the fabrication of previously presented OOCs.

In order to integrate the PEDOT:PSS electrodes in the Cytostretch, the fabrication process of the platform was improved by covering the TiN electrodes, previously developed by Pakazad et al. [25], with a Platinum (Pt) layer to allow the in situ electrochemical deposition of PEDOT:PSS [26]. The Pt electrodes were then coated with PEDOT:PSS and fully characterized with Scanning Electron Microscopy (SEM), Raman spectroscopy, Electrochemical Impedance Spectroscopy (EIS) and Cyclic Voltammetry (CV) in order to prove the PEDOT:PSS deposition and quantify the corresponding electrochemical impedance improvement.

\section{DeVICE FABrication}

The fabrication of the novel OOC equipped with lowimpedance PEDOT:PSS MEA, can be divided into two steps.

First, starting from the Cytostretch platform, an OOC with a Pt MEA is fabricated. The OOC consists of a cellculture environment in which a biological tissue grown and can be mechanically stimulated by inflating the stretchable PDMS membrane. The cells cultured in the OOC can be also monitored via the Pt electrodes directly embedded in the PDMS membrane. In order to fabricate this device, the wafer-scale microfabrication process previously presented by Pakazad et al. [25], [27] was modified and optimized. Second, the PEDOT:PSS is deposited directly on top of the Pt electrodes by electrochemical polymerization.

\section{A. OOC Equipped With Platinum MEA}

The wafer-scale fabrication of the new Cytostretch device with Pt-coated MEA is illustrated in Fig. 1. The process starts with the deposition of 2 and $6 \mu \mathrm{m}$ of silicon-dioxide $\left(\mathrm{SiO}_{2}\right)$ by plasma-enhanced chemical vapor deposition (PECVD) on the front and back of a 4" wafer, respectively. $\mathrm{The}^{\mathrm{SiO}_{2}}$ layer on the back is patterned by dry-etching to define the membrane area. The process continues by sputtering a $1.5 \mu \mathrm{m}$-thick aluminum (Al) layer on the front side of the wafer. The $\mathrm{Al}$ is then patterned by dry-etching to define the contact pads (Fig. 1(a)).

Next, a $100 \mathrm{~nm}$-thick Pt layer, preceded by a $20 \mathrm{~nm}$ of Ti as adhesion layer, is evaporated on the wafer and patterned by lift-off to form the electrodes of the MEA (Fig.1(a)). In the next step, the interconnection lines extending from the contact pads to the MEA are fabricated. For this, a $800 \mathrm{~nm}$-thick photosensitive polyimide (PI) (Fujifilm LTC 9305) layer is deposited by spin coating, patterned and cured at $350^{\circ} \mathrm{C}$ for 1 hour in low-pressure Nitrogen $\left(\mathrm{N}_{2}\right)$ atmosphere (Fig. 1(b)). Subsequently, a $200 \mathrm{~nm}$ of TiN, preceded by a $10 \mathrm{~nm}$ of $\mathrm{Ti}$ as adhesion layer, is sputtered on the front side of the wafer, and patterned by dry etching (Fig. 1(c)). A second layer of $800 \mathrm{~nm}$-thick PI is deposited and patterned to provide electrical insulation for the metal lines (Fig. 1(d)). An oxygen plasma treatment (Tepla Plasma 3000 - Power: $600 \mathrm{~W}$ Pressure: $250 \mathrm{ml} / \mathrm{min}$ - Time: $1 \mathrm{~min}$ ) is first performed to promote the adhesion between the two PI layers [28]. Subsequently, a Polydimethylsiloxane (PDMS) layer is deposited by spin coating on the front side of the wafer, at a spin speed of $6000 \mathrm{rpm}$ for 60 seconds and cured for $30 \mathrm{~min}$ at $90{ }^{\circ} \mathrm{C}$, resulting in a layer thickness of $10 \mu \mathrm{m}$. The adhesion of the PDMS to the PI was also improved by performing an 

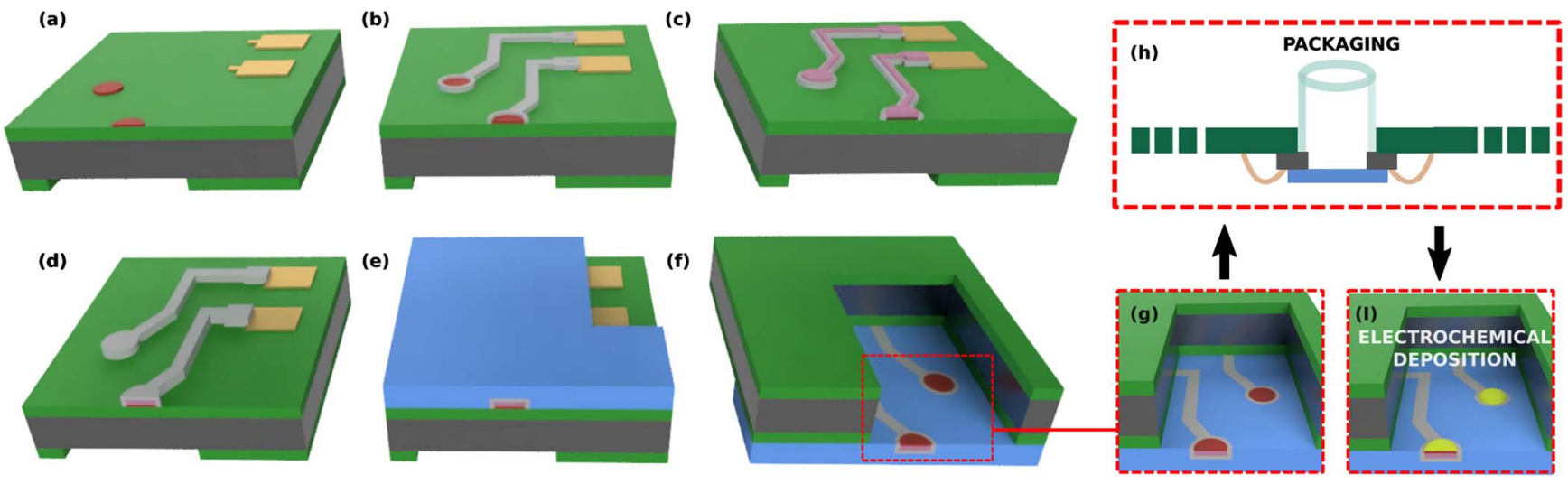

$\mathrm{Si} \mathrm{SiO}_{2} \quad$ PDMS

PI $\square$ TiN

Pt Al

PEDOT:PSS

Fig. 1. Process flow for the fabrication of OOC with PEDOT:PSS MEA: (a) contact pads and Platinum (Pt) electrodes are fabricated on top of a $2 \mu \mathrm{m}$ Silicon Oxide $\left(\mathrm{SiO}_{2}\right)$ layer. A $5 \mu \mathrm{m}$-thick SiO2 layer is deposited and patterned on the back of the wafer. (b) A $1 \mu \mathrm{m}$ layer of Polyimide (PI) is deposited and patterned. (c) The $200 \mathrm{~nm}$ thick Titanium Nitride (TiN) metal lines are fabricated. (d) The second layer of PI is deposited and patterned to isolate the metal lines. (e) A Polydimethylsiloxane (PDMS) layer is deposited and patterned to access the contact pads. (f,g) The PDMS membrane is released by etching the Si under the PDMS layer making the electrodes accessible. (h) The chips is then loaded, glued and wirebonded on a PCB. The packaging of the device ends by covering the chips with a glass cylinder. (i) PEDOT:PSS is deposited on the Pt electrodes via electrochemical polymerization.

argon plasma treatment (Trikon Sigma 204 - Power $120 \mathrm{~W}$ Pressure: $40 \mathrm{sccm}$ - Time: $15 \mathrm{sec}$ ), as previously reported in [29]. The contact pads are then opened by patterning the PDMS layer by means of reactive ion etching using an Al layer as hard mask (Fig. 1(e)). Finally, the membrane is released by removing the $\mathrm{Si}$ and the $\mathrm{SiO}_{2}$ layers underneath the membrane by means of deep reactive ion etching (DRIE) and buffered hydrofluoric acid (BHF), respectively (Fig. 1(g)).

The packaging procedure starts by dicing the 4-inch wafer with an automatic dicing saw. More than 100 Cytostretch chips can be obtained out of each wafer. Four chips are mounted on a PCB with a fully-automatic pick-and-place system. The PCB is designed to fit into a MultiChannel System in vitro recording devices (MEA2100-System). The Al contact pads on the chip are subsequently wire-bonded to the PCB. The packaging is then finished by mounting a glass cylinder on top of each dice on the PCB with epoxy glue (EPO-TEK 353ND-T) cured at $75{ }^{\circ} \mathrm{C}$ for 90 minutes at atmospheric pressure. This glass cylinder creates an open well structure on top of each chip, where the cells can be seeded. The PDMS membrane can be then stretched by connecting the OOC to an external system that applies pneumatic pressure to the back of the device.

In this work, this well is going to be used as the electrochemical cell for the PEDOT:PSS deposition on top of the Pt electrodes embedded in the stretchable membrane of the Cytostretch. The well can be also directly molded on top of the chips by an automated and monolithic molding technique as previously reported in [30].

\section{B. PEDOT Electrochemical Deposition}

The next step is to cover the Pt electrodes of the Cytostretch MEA with a PEDOT:PSS layer (Fig. 1(h)). The in-situ deposition of PEDOT:PSS coating was performed from an aqueous solution containing a mixture of $0.001 \mathrm{M}$ of EDOT monomers, 0.1 M of Sodium Poly(Styrene Sulfonate) (NaPSS,
$\mathrm{M}_{\mathrm{w}}:$ 70.000) as the dopant material as well as a surfactant, and $0.005 \mathrm{M}$ of Tetrabutylammonium Tetrafluoroborate $\left(\mathrm{TBABF}_{4}\right)$ as secondary doping and to increase the conductivity of the solution. The electrochemical procedures in this study were performed with an Autolab Potentiostat (Metrohm), with a platinum strip and a miniaturized $\mathrm{Ag} / \mathrm{AgCl}$ (supplied by EDAQ) as the counter and reference electrodes, respectively.

The cyclic voltammetry (CV) deposition method with a three electrodes cell setup [27], was utilized in order to increase the deposition rate by exploiting the capacitive current. In this procedure, the voltage on the working electrode is scanned over a $0.2-1.3 \mathrm{~V}$ (vs. $\mathrm{Ag} / \mathrm{AgCl}$ ) range with a scan rate of $0.5 \mathrm{~V} / \mathrm{s}$. The amount of charge delivered during the deposition is $3 \mu \mathrm{C}$.

After each deposition, the MEA was drained of the remaining solution and rinsed with DI water. Afterwards, vacuum drying was performed at room temperature for 30 minutes to remove all liquid residues.

\section{MEA Characterization Procedure}

The deposition of the PEDOT:PSS was verified by comparing the SEM images of the electrodes before and after the electrochemical deposition and by performing Raman spectroscopy on the electrodes. This analysis was performed with a Renishaw inVia Raman Spectroscope, with $488 \mathrm{~nm}$ wavelength and 300 to $1900 \mathrm{~cm}^{-1}$ spectra coverage.

The performance of the MEA is investigated by means of electrochemical characterization, namely EIS to determine the impedance of the MEA over a certain frequency range and $\mathrm{CV}$ measurements to estimate the amount of charge that can be delivered within a certain voltage scan.

The improvement provided by the PEDOT:PSS was measured by characterizing the electrodes before and after the electrochemical deposition of the polymer. The performance of the Pt and PEDOT:PSS electrodes were also compared to 

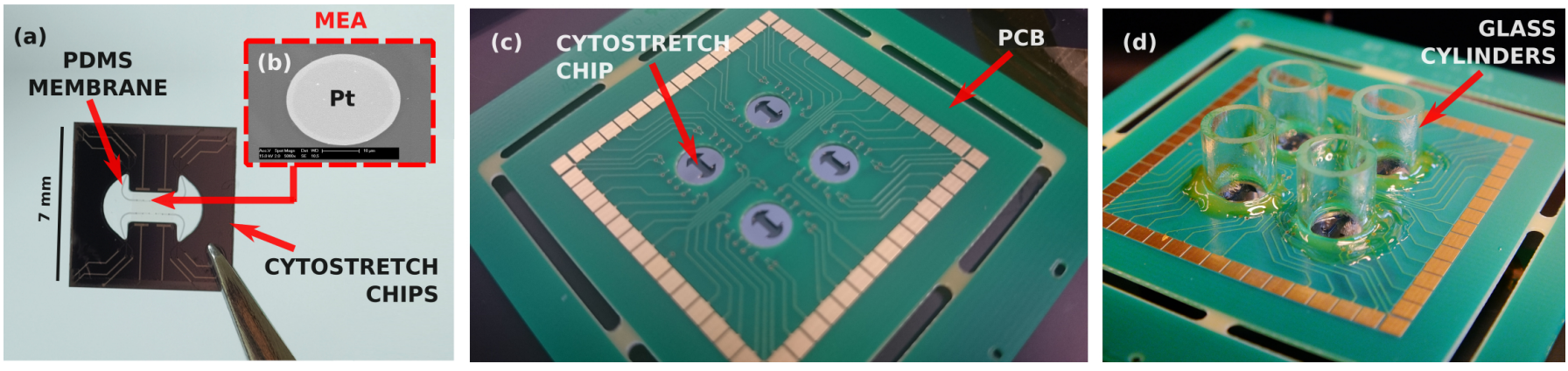

Fig. 2. (a) Optical image of one Cytostretch chips including the Pt MEA embedded in a stretchable PDMS membrane. The chip includes 12 Pt circular electrodes (diameter: $30 \mu \mathrm{m}$, pitch $100 \mu \mathrm{m}$ ). (b) SEM image of Pt MEA. (c,d) Optical image of the multi-well plate consisting of four Cytostretch chips mounted on a PCB before (c) and after (d) attaching the glass cylinders on the chips.
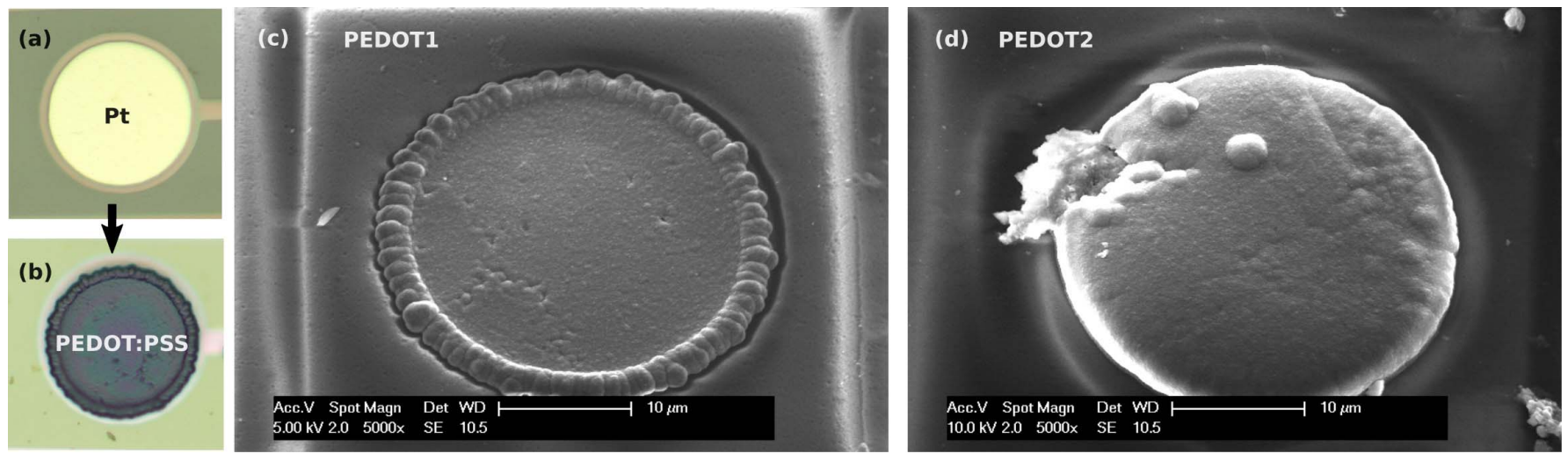

Fig. 3. (a) Optical image of one of the electrodes embedded in a stretchable PDMS membrane before (a) and after (b) the electrochemical deposition of PEDOT:PSS. (c) SEM image of the PEDOT:PSS electrode deposited with CV deposition, scanning the voltage on the working electrode between $0.2-1.3 \mathrm{~V}$ (vs. $\mathrm{Ag} / \mathrm{AgCl}$ ) with a scan rate of $0.5 \mathrm{~V} / \mathrm{s}$ (PEDOT1). (d) SEM image of the PEDOT:PSS electrode deposited with CV deposition, scanning the voltage on the working electrode between $0.2-1 \mathrm{~V}(\mathrm{vs}$. Ag/AgCl) with a scan rate of $0.5 \mathrm{~V} / \mathrm{s}$ (PEDOT2).

standard TiN electrodes included in the previous version of the Cytostretch [11].

All the electrochemical characterization procedures were done with a three electrodes setup [27] in Phosphate Buffered Saline (PBS) medium (by Sigma Aldrich). The electrochemical characterization, i.e. EIS and CV measurements, was performed in a single run for each microelectrode type without changing the PBS medium.

The EIS measurements in this study were performed in the frequency range of $0.01 \mathrm{~Hz}-10 \mathrm{kHz}$, AC signal with an amplitude of $50 \mathrm{mV}$ (RMS). The $\mathrm{CV}$ measurements were performed over a voltage range of $-0.6 \mathrm{~V}$ to $0.8 \mathrm{~V}$, which also corresponds to the electrochemical window of water [31]. The starting potential was $0 \mathrm{~V}$, with the scan rate of $0.5 \mathrm{~V} / \mathrm{s}$ (forward scan first). Each measurement consisted of 5 repetitive scans.

\section{Results And Discussion}

Fig. 2 shows a successfully fabricated OOC device with Pt electrodes before being mounted on the PCB. The SEM image (top view) of one of the Platinum electrodes (with an area of $700 \mu \mathrm{m}^{2}$ ) embedded in the stretchable membrane is displayed in Fig.2b. The Ti layer in between the TiN the Pt layers appears to have successfully promote the adhesion, since none of the fabricated electrodes indicated any Pt detachment after the membrane release, as confirmed by SEM analysis. The chips mounted and wirebonded on the PCB and the glass cylinders glued on the PCB are shown in Fig. 2(c) and (d), respectively.

The deposition of a PEDOT:PSS layer on top of the Pt electrodes was successfully achieved. The material was easily identified on the electrodes by the change of color observed on the Pt electrodes (Fig. 3(a,b)) in agreement with what previously reported [26], [32] and confirmed by SEM imaging. The SEM images of two PEDOT:PSS electrodes are shown in Fig. 3(d,e). The PEDOT:PSS was deposited only on the exposed electrodes, proving that the adhesion between the two layers of PI, was sufficient to prevent any current leakage during the deposition. The glass well and the PEDOT:PSS MEA at the bottom were rinsed 5 times with PBS using a pipette. No PEDOT:PSS peeling off was detected, proving the adhesion of the material to the electrodes.

The results of the Raman spectroscopy measurements on the PEDOT:PSS coated microelectrodes are shown in Fig. 4. The Raman spectrum presents a similar trend with the results observed by Kayinamura et al. [26] and Łapkowski and Proń [32]. The maximum peak appears at $1436 \mathrm{~cm}-1$ that corresponds to the symmetric vibration of $\mathrm{C} \alpha=\mathrm{C} \beta$ bond in the oxidized polymer chain [26]. Several minor peaks at around 


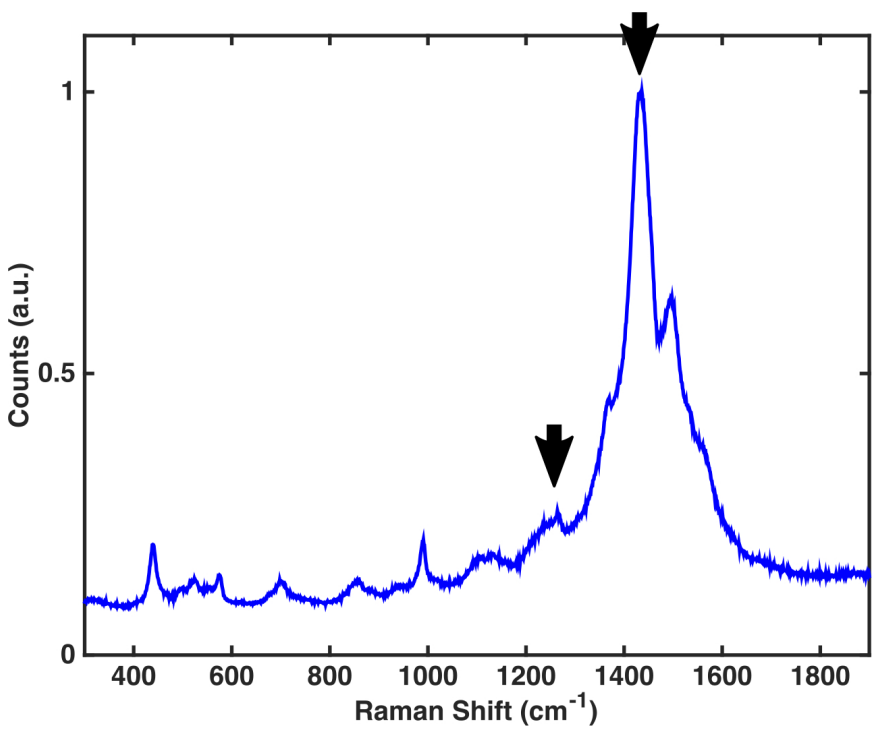

Fig. 4. Raman spectra of PEDOT:PSS grown on the Pt electrodes showing a similar trend with the results observed by Kayinamura et al. [26] and Łapkowski and Proń [32]. The maximum peak appears at $1436 \mathrm{~cm}^{-1}$ and several minor peaks can be seen around $1249 \mathrm{~cm}^{-1}$.

$1249 \mathrm{~cm}-1$ are also visible, which correspond to the $\mathrm{C} \alpha-\mathrm{C} \alpha$ bond in the doped PEDOT:PSS polymer [32]. These results confirm the successful deposition of the PEDOT:PSS film.

During the CV deposition, charge response (Fig. 5(a)) and current response (Fig. 5(b)) were recorded. Only five of the CV cycles are reported in Fig. 5(b) (Cycle 01,20,40,60 and 80). As can be inferred by the current response (Fig. 5(a) in blue), the deposition rate of the PEDOT:PSS was not constant during the process. In fact, the charge delivered by the electrode during the deposition shows an abrupt increase in the first cycles. This phenomenon is due to the high current peaks caused by the PEDOT:PSS oxidation [33]-[35], appearing between 1 and $1.3 \mathrm{~V}$ in the current response graphs of the first few cycles. This can be seen in the first deposition (Cycle 01) reported in Fig. 5.(b). As previously presented by Kayinamura et al., [26] a high deposition rate can cause rapid movement of ions in the solution, which results in a less ordered polymer chain and eventually in a high impedance. In order to avoid these current peaks while minimizing the deposition time, the potential range was narrowed down to $0.2-1 \mathrm{~V}$ (vs. $\mathrm{Ag} / \mathrm{AgCl}$ ). The current response (Fig. 5(a) in red) and the charge delivery plot (Fig. 5(c)) after this optimization, confirmed that the deposition was in fact occurring at an almost constant deposition rate in the first few cycles, avoiding any current peaks. Moreover, the deposition rate was progressively increasing, due to the increasing current following the decrease of the electrode impedance.

The EIS and CV measurements results are shown in Fig. 6. A significant change was observed in both the impedance and the phase characteristics of the MEA after PEDOT:PSS deposition. The control TiN MEA and Pt MEA exhibit at $1 \mathrm{kHz}$ a $1.3 \pm 670 \mathrm{M} \Omega$ and $865 \pm 35 \mathrm{k} \Omega$ impedance, respectively. The impedance decrease is different for each deposition condition. The deposition with potential range between 0.2 and $1.3 \mathrm{~V}$ (referred here as PEDOT1) yields a
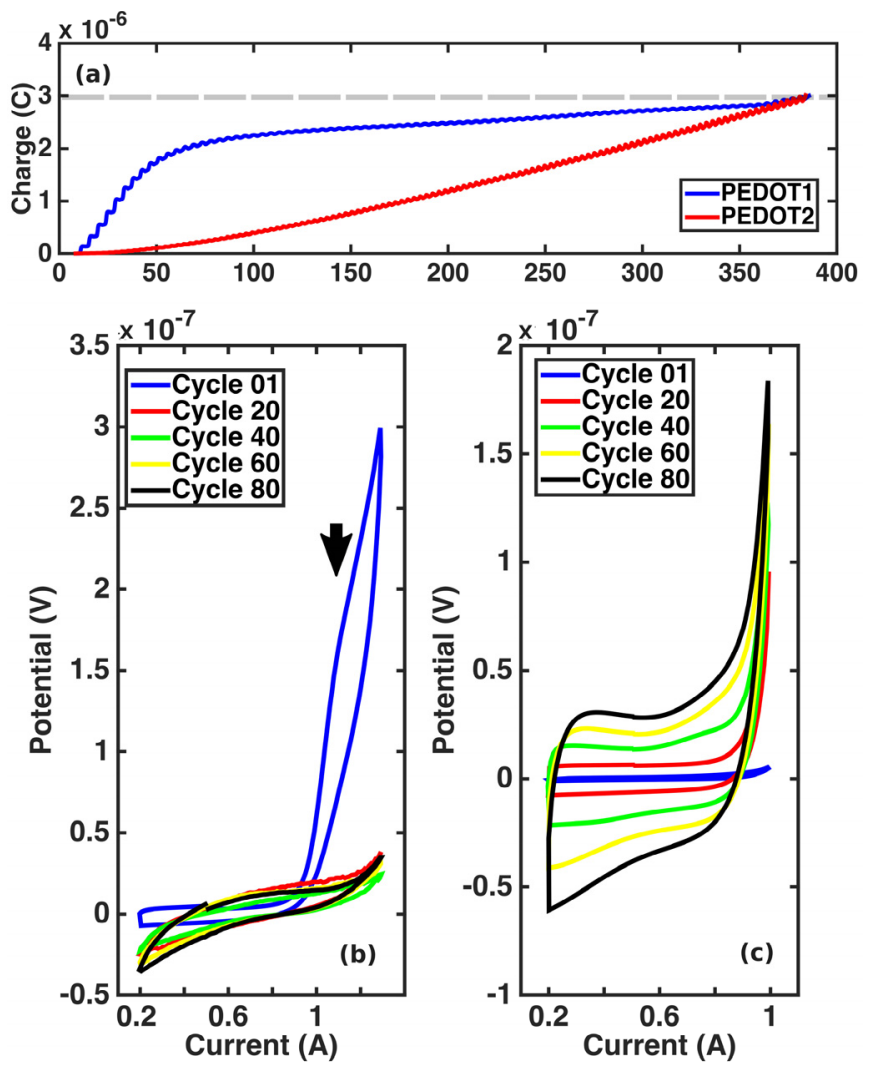

Fig. 5. (a) Charge delivered through the electrode during of PEDOT1 (in blue) and PEDOT2 (in red) depositions. (b) Current response during cycle $01,20,40,60$ and 80 for PEDOT1 deposition. (c) Current response during cycle $01,20,40,60$ and 80 for PEDOT2 deposition.

small improvement in term of the electrochemical impedance $(705 \pm 21 \mathrm{k} \Omega 1 \mathrm{kHz}$,), while the PEDOT:PSS deposited with a potential rate between 0.2 and $1 \mathrm{~V}$ (PEDOT2) significantly reduces the impedance, reaching a value of $59 \pm 2 \mathrm{k} \Omega$, which is in the same range of PEDOT:PSS electrodes integrated in non stretchable surfaces [20]. This corresponds to a $95.5 \%$ and a 93\% of impedance reduction when compared to TiN and $\mathrm{Pt}$, respectively. The phase of PEDOT2 showed also a more ohmic behavior for frequencies higher than $100 \mathrm{~Hz}$.

When compared to TiN and $\mathrm{Pt}$, the improvement of the electrochemical impedance and the change of phase characteristics obtained with the PEDOT:PSS coating are due to the inherent faradaic activities of the PEDOT:PSS film, attributed to the faradaic doping/undoping reactions, which allow ions to react at a faster rate [36], [37]. This value is even higher than the value reported in earlier studies [38], proving that a slow and ordered deposition of the PEDOT:PSS can improve even further the performances of this material.

The significant difference between PEDOT1 and PEDOT2 characteristics is mainly caused by the different deposition rate and consequently by the orderly structures among the polymer chains.

The CV curves from each of the microelectrodes types are shown in Fig. 6.(c). The CV of PEDOT:PSS coating, particularly PEDOT2, shows very large capacitive current, compared to TiN and Pt (Fig.6.(a)), both in the forward and the reverse scans. This is caused by the large double layer 

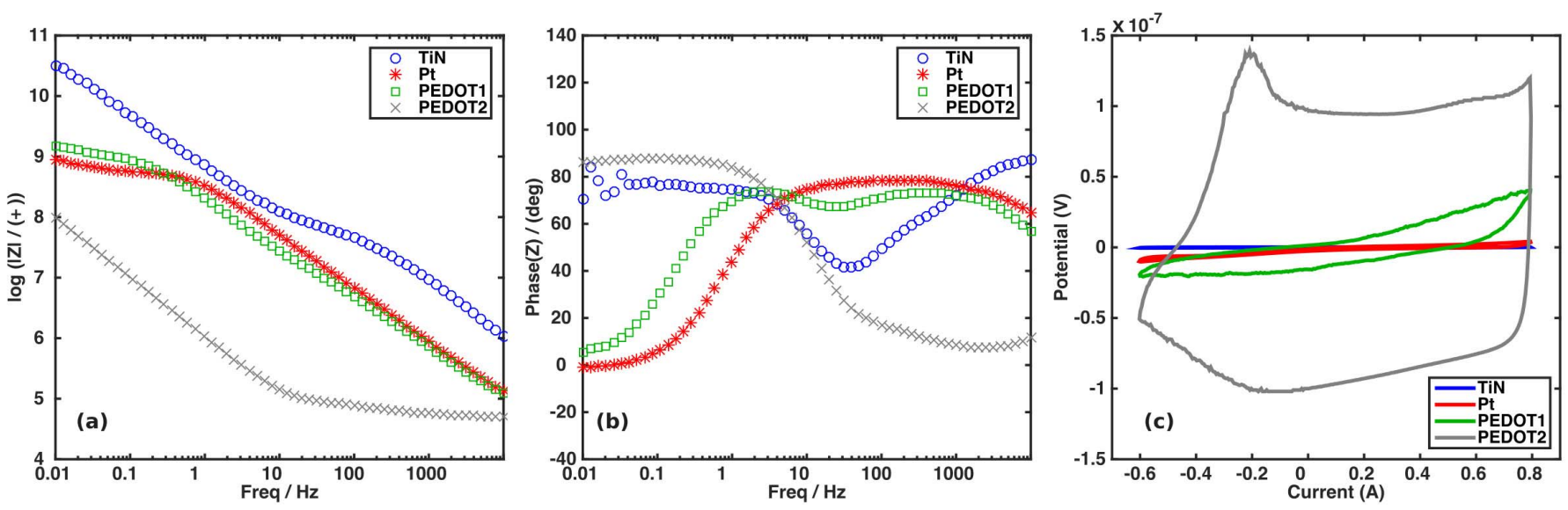

Fig. 6. (a,b) Bode plot ((a) amplitude and (b) phase) of impedance spectra of a TiN electrode, a Pt electrode and two PEDOT:PSS electrodes (deposited with PEDOT1 and PEDOT2 programs) ( $30 \mu \mathrm{m}$ diameter). EIS at $1 \mathrm{kHz}$ are equal to $9.32 \mathrm{M} \Omega, 875 \mathrm{k} \Omega, 720 \mathrm{k} \Omega$ and $58 \mathrm{k} \Omega$ for TiN, Pt, PEDOT1 and PEDOT2 electrodes, respectively. (c) CV measurement from four types of the microelectrodes combined in one graph.

TABLE I

Charge Delivery CAPACity (CDC)

\begin{tabular}{lcc}
\hline \hline Material & $\begin{array}{c}\mathrm{CDCa} \\
\left(\mathrm{mC} / \mathrm{cm}^{2}\right)\end{array}$ & $\begin{array}{c}\mathrm{CDCc} \\
\left(\mathrm{mC} / \mathrm{cm}^{2}\right)\end{array}$ \\
\hline TiN & 0.055 & 0.095 \\
Pt & 0.364 & 0.532 \\
PEDOT:PSS & 30.88 & 33.45 \\
(PEDOT2) & & \\
\hline \hline
\end{tabular}

CDCa: Anodic Charge Delivery Capacity.

CDCc: Cathodic Charge Delivery Capacity

capacitance of the PEDOT:PSS coating. This proves once again that PEDOT:PSS provides a better performance than TiN and Pt in the Cytostretch MEA. Furthermore, the charge delivery capacity (CDC) value for each microelectrode type was derived from this measurement to qualitatively evaluate the microelectrode surface area. The values of CDC are calculated using (1), separately for the cathodic (below $0 \mathrm{~A}$ ) and anodic scans and listed in Table. 1.

$$
C D C=\frac{1}{v \times A} \int_{E c}^{E a}[I] \cdot d E
$$

where $I$ is the measured current (A), $v$ is the scan rate $(\mathrm{V} / \mathrm{s})$, $A$ is the area of the electrode $\left(\mathrm{cm}^{2}\right), E_{a}$ and $E_{c}$ are the peak anodic and cathodic potential, respectively [39]. The CDC values of PEDOT:PSS coated microelectrode are 3 and 2 orders of magnitude higher than those of the TiN and Pt microelectrode, respectively, proving once again the increase of the surface area of the microelectrode. This result confirms the feasibility of integrating PEDOT:PSS coated microelectrodes into stretchable OOC, which also provides superior electrochemical performance compared to the more conventional Pt and TiN microelectrodes.

\section{CONCLUSION}

This work introduces the fabrication, characterization and optimization of the first stretchable $\mathrm{OOC}$ equipped with a PEDOT:PSS coated MEA. In order to fabricate this device, the modular OOC platform, known as Cytostretch, was employed as starting point.

The fabrication of the OOC was successfully improved by coating its TiN MEA with a Pt layer in order to guarantee the adhesion between the PEDOT:PSS and the electrodes. The optimized Cytostretch devices were then packaged and assembled on a PCB. After that, the conducting polymer was locally deposited on the electrodes. The deposition was verified by means of optical and SEM imaging. Moreover, the material was analyzed with Raman spectroscopy, and the results compared to previous work so to prove that the deposited material was indeed PEDOT:PSS.

The electrochemical performance of the MEA embedded in the novel device was characterized before and after the PEDOT:PSS deposition, and compared to the previous presented version of the Cytostretch (equipped with a TiN MEA). The PEDOT:PSS electrodes showed an electrochemical impedance reduction of 99.4 and $93.3 \%$ when compared to TiN and Pt electrodes. Moreover, the CV characterization of PEDOT:PSS electrodes confirmed a higher capacitive current, compared to TiN and Pt. In fact, the CDC values for the PEDOT:PSS electrodes were 2 and 3 orders of magnitude higher that the $\mathrm{Pt}$ and TiN electrodes, respectively. The impedance might be further optimized by tuning thickness and deposition rate.

This work is a first step toward the integration of lowimpedance PEDOT:PSS MEA in an OOC, that could eventually allows low-noise and on-line monitoring of cells in these in vitro models and promote their use in cardiotoxicity tests during the preclinical phase of medicine development.

\section{ACKNOWLEDGMENT}

The authors gratefully acknowledge the technical support and advice of the staff at the TUD-Else Kooi Lab and the Institute for human Organ and Disease Model Technologies (hDMT).

\section{REFERENCES}

[1] N. Beißner, T. Lorenz, and S. Reichl, "Organ on chip," in Microsystems for Pharmatechnology Manipulation of Fluids, Particles, Droplets, and Cells. Cham, Switzerland: Springer, 2016, pp. 299-339. 
[2] A. D. Van der Meer and A. Van den Berg, "Organs-on-chips: Breaking the in vitro impasse," Integr. Biol., vol. 4, pp. 461-470, May 2012.

[3] A. Van de Stolpe and J. Den Toonder, "Workshop meeting report Organs-on-Chips: Human disease models," Lab Chip, vol. 13, no. 18, pp. 3449-3470, Mar. 2013.

[4] W. F. Quirós-Solano et al., "Microfabricated tuneable and transferable porous PDMS membranes for Organs-on-Chips," Sci. Rep, vol. 8, no. 1, p. 13524, Sep. 2018.

[5] V. van Duinen, S. J. Trietsch, J. Joore, P. Vulto, and T. Hankemeier, "Microfluidic 3D cell culture: From tools to tissue models," Current Opinion Biotechnol., vol. 35, pp. 118-126, Dec. 2015.

[6] Y. B. Arik et al., "Barriers-on-chips: Measurement of barrier function of tissues in organs-on-chips," Biomicrofluidics, vol. 12, no. 4, Jun. 2018, Art. no. 042218.

[7] S. F. Cogan, "Neural stimulation and recording electrodes," Аnnи. Rev. Biomed. Eng., vol. 10, pp. 275-309, Aug. 2008.

[8] T. M. Pearce, J. A. Wilson, S. G. Oakes, S.-Y. Chiu, and J. C. Williams, "Integrated microelectrode array and microfluidics for temperature clamp of sensory neurons in culture," Lab Chip, vol. 5, no. 1, pp. 97-101, 2005.

[9] A. Scott, K. Weir, C. Easton, W. Huynh, W. J. Moody, and A. Folch, "A microfluidic microelectrode array for simultaneous electrophysiology, chemical stimulation, and imaging of brain slices," Lab Chip, vol. 13, no. 4, pp. 527-535, 2013.

[10] B. M. Maoz et al., "Organs-on-chips with combined multi-electrode array and transepithelial electrical resistance measurement capabilities," Lab Chip, vol. 17, no. 13, pp. 2294-2302, 2017.

[11] N. Gaio et al., "Cytostretch, an organ-on-chip platform," Micromachines, vol. 7, no. 7, p. 120, Jul. 2016.

[12] S. Hui, C. Liu, Q. Jiang, and J. Xu, "Effective approaches to improve the electrical conductivity of PEDOT:PSS: A review," Adv. Electron. Mater, vol. 1, no. 4, Mar. 2015, Art. no. 1500017.

[13] D. J. Lipomi, J. A. Lee, M. Vosgueritchian, B. C.-K. Tee, J. A. Bolander, and Z. Bao, "Electronic properties of transparent conductive films of PEDOT: PSS on stretchable substrates," Chem. Mater. vol. 24, pp. 373-382, Dec. 2012.

[14] T. D. Y. Kozai et al., "Chronicin vivo evaluation of PEDOT/CNT for stable neural recordings," IEEE Trans. Biomed. Eng., vol. 63, no. 1, pp. 111-119, Jan. 2016

[15] A. Schander, T. Teßmann, S. Strokov, H. Stemmann, A. K. Kreiter, and W. Lang, "In-vitro evaluation of the long-term stability of PEDOT:PSS coated microelectrodes for chronic recording and electrical stimulation of neurons," in Proc. 38th Annu. Int. Conf. IEEE Eng. Med. Biol. Soc. (EMBC), Aug. 2016, pp. 6174-6177.

[16] S. Savagatrup et al., "Plasticization of PEDOT:PSS by common additives for mechanically robust organic solar cells and wearable sensors," Adv. Funct. Mater, vol. 25, no. 3, pp. 427-436, Jan. 2015.

[17] Y. Galagan et al., "Scaling up ITO-free solar cells," Adv. Energy Mater., vol. 4, no. 2, Jan. 2014, Art. no. 1300498.

[18] R. Kim, S. Joo, H. Jung, N. Hong, and Y. Nam, "Recent trends in microelectrode array technology for in vitro neural interface platform," Biomed. Eng. Lett., vol. 4, no. 2, pp. 129-141, Jun. 2014.

[19] T. Meyer, C. Leisgen, B. Gonser, and E. Günther, "QT-screen: Highthroughput cardiac safety pharmacology by extracellular electrophysiology on primary cardiac myocytes," Assay Drug Dev. Technol., vol. 2, no. 5, pp. 507-514, Nov. 2004.

[20] R. Gerwig et al., "PEDOT-CNT composite microelectrodes for recording and electrostimulation applications: Fabrication, morphology, and electrical properties," Front. Neuroeng., vol. 5, p. 8, May 2012.

[21] L. Peng and F. Yan, "Organic thin-film transistors for chemical and biological sensing," Adv. Mater., vol. 24, no. 1, pp. 34-51, Jan. 2011.

[22] D. Nilsson, T. Kugler, P. O. Svensson, and M. Berggren, "An all-organic sensor-transistor based on a novel electrochemical transducer concept printed electrochemical sensors on paper,' Sensors Actuators B, Chem., vol. 86, nos. 2-3, pp. 193-197, 2002.

[23] K. Krishnamoorthy, R. S. Gokhale, A. Q. Contractor, and A. Kumar, "Novel label-free DNA sensors based on poly $(3,4-$ ethylenedioxythiophene)," Chem. Commun., vol. 7, pp. 820-821, Feb. 2004.

[24] P. Lin, F. Yan, J. Yu, H. L. W. Chan, and M. Yang, "The application of organic electrochemical transistors in cell-based biosensors," Adv. Mater, vol. 22, no. 33, pp. 3655-3660, Sep. 2010.

[25] S. K. Pakazad, A. Savov, A. van de Stolpe, and R. Dekker, "A novel stretchable micro-electrode array (SMEA) design for directional stretching of cells," J. Micromech. Microeng, vol. 24, no. 3, Feb. 2014, Art. no. 034003
[26] Y. P. Kayinamura, M. Ovadia, D. Zavitz, and J. F. Rubinson, "Investigation of near ohmic behavior for poly(3,4-ethylenedioxythiophene): A model consistent with systematic variations in polymerization conditions," ACS Appl Mater Inter, vol. 2, no. 9, pp. 2653-2662, Aug. 2010.

[27] N. Gaio et al., "Upside-down Carbon nanotube (CNT) micro-electrode array (MEA)," in Proc. IEEE SENSORS, Nov. 2015, pp. 1-4.

[28] Y. Nakamura, Y. Suzuki, and Y. Watanabe, "Effect of oxygen plasma etching on adhesion between polyimide films and metal," Thin Solid Films, vols. 290-291, pp. 367-369, Dec. 1996.

[29] S. Joshi, A. van Loon, A. Savov, and R. Dekker, "Adhesion improvement of polyimide/PDMS interface by polyimide surface modification," MRS Adv., vol. 1, no. 1, pp. 33-38, Jan. 2016.

[30] N. Gaio et al., "A multiwell plate Organ-on-Chip (OOC) device for in-vitro cell culture stimulation and monitoring," in Proc. IEEE Micro electro mech. Syst. (MEMS), Jan. 2018, pp. 314-317.

[31] T. L. Rose and L. S. Robblee, "Electrical stimulation with Pt electrodes. VIII. Electrochemically safe charge injection limits with $0.2 \mathrm{~ms}$ pulses (neuronal application)," IEEE Trans. Biomed. Eng., vol. 37, no. 11, pp. 1118-1120, Nov. 1990.

[32] M. Łapkowski and A. Pron, "Electrochemical oxidation of poly(3,4ethylenedioxythiophene)__ 'in situ' conductivity and spectroscopic investigations," Synth. Metals, vol. 110, no. 1, pp. 79-83, Mar. 2000.

[33] Allen J. Bard and Larry R. Faulkner, Electrochemical Methods: Fundamentals and Applications, 2nd ed. New York, NY, USA: Wiley, 2001.

[34] G. A. Mabbott, "An introduction to cyclic voltammetry," J. Chem. Educ., vol. 60 , no. 9, p. 697, 1983.

[35] H. Yamato, M. Ohwa, and W. Wernet, "Stability of polypyrrole and poly(3,4-ethylenedioxythiophene) for biosensor application," J. Electroanal. Chem., vol. 397, nos. 1-2, pp. 163-170, Nov. 1995.

[36] H. J. Ahonen, J. Lukkari, and J. Kankare, "n- and p-Doped Poly(3,4-ethylenedioxythiophene): Two electronically conducting states of the polymer," Macromolecules, vol. 33, no. 18, pp. 6787-6793, Aug. 2000.

[37] J. Bobacka, A. Lewenstam, and A. Ivaska, "Electrochemical impedance spectroscopy of oxidized poly(3,4-ethylenedioxythiophene) film electrodes in aqueous solutions," J. Electroanal. Chem, vol. 489, nos. 1-2, pp. 17-27, Jul. 2000.

[38] Z. A. King, C. M. Shaw, S. A. Spanninga, and D. C. Martin, "Structural, chemical and electrochemical characterization of poly $(3,4$ Ethylenedioxythiophene) (PEDOT) prepared with various counterions and heat treatments," Polymer, vol. 52, no. 5, pp. 1302-1308, Mar. 2011.

[39] E. Slavcheva, R. Vitushinsky, W. Mokwa, and U. Schnakenberg, "Sputtered iridium oxide films as charge injection material for functional electrostimulation," J. Electrochem. Soc., vol. 151, no. 7, pp. E226-E237, Jul. 2004.

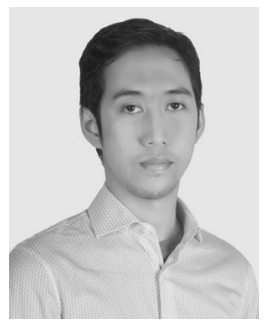

Affan K. Waafi received the B.S. degree in engineering physics from the Institut Teknologi Bandung, Bandung, Indonesia, in 2014, and the M.Sc. (cum laude) degree in biomedical engineering from the Delft University of Technology, Delft, The Netherlands, in 2018.

He was involved in the development of the Cytostretch device as part of his master thesis project with the Delft University of Technology. His current research interests are mainly related with electronic-tissue interface, biomedical electronic device, bio-MEMS, and conductive polymer materials. Previously, he was involved in the development of the brain-computer Interface platform during his undergraduate study, also authored or coauthored several conference publications from this research. 


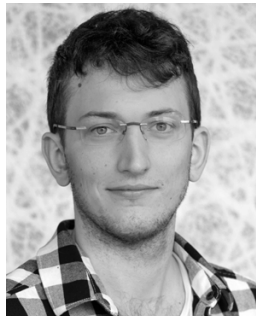

Nikolas Gaio received the B.S. degree in electronic engineering from the Polytechnic University of Milan, Milan, Italy, in 2012, the B.Eng. degree in electronic information from Tongji University, Shanghai, China, in 2013, and the M.Sc. (cum laude) degree in biomedical engineering from the Faculty of Mechanical, Maritime, and Materials Engineering, TU Delft, in 2015, where he is currently pursuing the Ph.D. degree.

In 2011, he was a Visiting Student at Tongj University, Shanghai. In 2013, he joined as a Guest Researcher with the Shenzhen Institute of Advanced Technology, Chinese Academy of Science. His research focuses on organon-chip and stretchable microelectrode arrays. His areas of interests include design, microfabrication, and characterization of MEMS devices. He received the Best Student Paper Award from the 2015 IEEE Sensors Conference, the Public Poster Award from the ICT Open Conference in 2016, and the Lush Prize (Young Researcher) in 2018. He is the Founder of BI/OND (BIOND Solution B.V.). BI/OND provides dynamic cell culture environments to recreate human physiology and pathology for predictive in vitro assays.

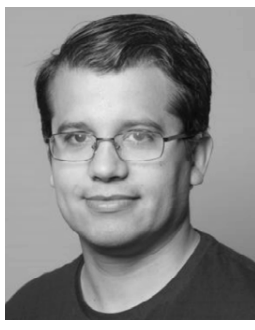

William F. Quiros-Solano received the B.S degree in electronics engineering from the Instituto Tecnológico de Costa Rica, Cartago, Costa Rica, in 2011, and the M.Sc. (cum laude) degree in electronics engineering with emphasis on microelectromechanical systems (MEMS) in 2014 $\mathrm{He}$ is currently pursuing the Ph.D. degree with the Department of Microelectronics, Delft University of Technology (TU Delft). In 2011, he joined the School of Electronic Engineering as a Research and Teaching Assistant. His research focuses on organ-on-chip with particular interest in design, fabrication, and characterization of novel microstructures for cell culture microenvironments. He received a Scholarship from the Instituto Tecnológico de Costa Rica to pursue his Ph.D. degree.

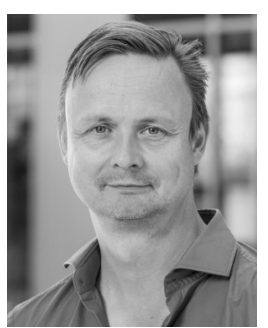

Paul Dijkstra received the M.S. degree in chemical engineering from the Eindhoven University of Technical, Eindhoven, The Netherlands, in 1992. $\mathrm{He}$ is currently a Senior Architect of Micro Assembly with the Department of Micro Devices, Philips Innovation Services. He has 25 years of experience in assembly technologies in the field of electronic package innovation and micro device assembly. His current works focus on the development of new emerging applications in areas like photonics, in and on body devices, and MEMS packages.

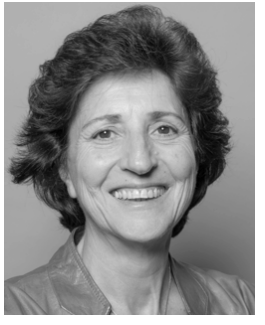

Pasqualina M. Sarro (M'83-SM'97-F'07) received the Laurea (cum laude) degree in solid-states physics from the University of Naples, Italy, in 1980, and the Ph.D. degree in electrical engineering from the Delft University of Technology, The Netherlands, in 1987. From 1981 to 1983, she was a Postdoctoral Fellow in the Division of Engineering, Photovoltaic Research Group, Brown University, Providence, RI, USA. She joined the Faculty of Electrical Engineering, Delft University of Technology, to establish and lead research on silicon micromachining, integrated sensor, MEMS, and material processing. In 2001, she was appointed the Antoni van Leeuwenhoek Full Professor for research merits. She has coauthored more than 500 publications. Her main research interests include novel materials and structures for MEMS and NEMS to be applied in health, automotive, environmental applications, and scientific instrumentation. She received several awards (elected member KNAW; IEEE Sensor Meritorious Award, Eurosensors Fellow, IEEE Bosch Award). She has co-chaired many international conferences in the field of sensors and MEMS. She was an Associate Editor of the IEEE SENSORS JOURNAL from 2006 to 2009 and IEEE JMEMS from 2014 to 2020.

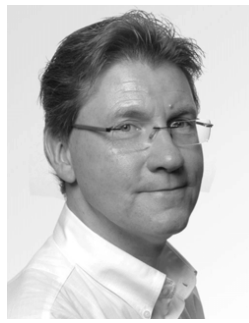

Ronald Dekker received the M.Sc. degree in electrical engineering from the Technical University of Eindhoven and the Ph.D. degree from the Technical University of Delft.

He joined Philips Research in 1988, where he worked on the development of RF technologies for mobile communication. Since 2000, his focus shifted to the integration of complex electronic sensor functionality on the tip of the smallest minimal invasive instruments, such as catheters and guide-wires. In 2007, he was appointed to part time Professor with the Technical University of Delft, with a focus on organ-on-chip and bioelectronics medicines. He has published many leading journals and conferences and holds more than 60 patents. 